\title{
Successful treatment of a giant exposed blood ves- sel in a gastric ulcer by endoscopic sclerotherapy with N-butyl-2-cyanoacrylate
}
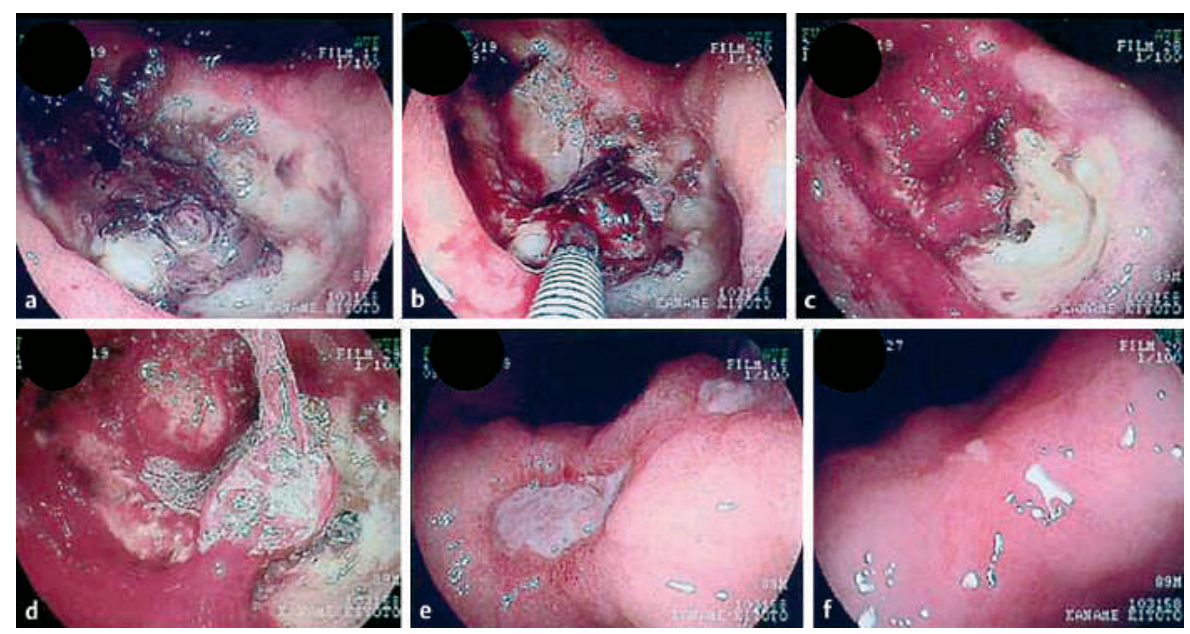

Figure 1 Endoscopic views of a giant exposed blood vessel in a gastric ulcer treated by endoscopic sclerotherapy with N-butyl-2-cyanoacrylate. The patient's bleeding was seen to be coming from a giant exposed blood vessel (a). Ethanol was injected into the navel-like region in the center of the ulcer (b), but pulsatile bleeding began from the injection point after the needle was removed (c). We decided to use N-butyl-2-cyanoacrylate for sclerotherapy: polymer was observed to form on the surface of the blood vessel and the pulsatile bleeding finally stopped 5 minutes after the injection (d). Follow-up endoscopy indicated that regenerative mucosa was growing around the ulcer and that the ulcer was gradually diminishing in size $(\mathbf{e}, \mathbf{f})$.

An 80-year-old man was admitted with hematemesis. Endoscopic examination revealed a deep giant gastric ulcer, and bleeding from the exposed blood vessel was detected in the lesser curvature of the angles of the stomach (Figure $\mathbf{1} \mathbf{a}$ ). We thought that only the navel-like region in the center of the ulcer was an exposed blood vessel, and ethanol was injected into the edge of this region (Figure $\mathbf{1} \mathbf{b})$. However, after the needle was removed, pulsatile bleeding began from the injection point (Figure 1 c), revealing that the wider area around the navel-like region was also part of the giant exposed blood vessel, which measured $10 \mathrm{~mm}$ (using biopsy forceps).

We decided to use N-butyl-2-cyanoacrylate. Immediately after injecting a small amount of $50 \%$ glucose, $0.5 \mathrm{~mL}$ of stock solution of N-butyl-2-cyanoacrylate (Histoacryl; Aesculap AG \& Co., Tuttlingen, Germany) was injected into the center of the exposed blood vessel, followed by further injection of a small amount of 50\% glucose. The formation of polymer was observed on the surface of the blood vessel and the pulsatile bleeding finally stopped 5 minutes after the injection of N-butyl-2-cyanoacrylate (Figure 1 d). Follow-up endoscopy indicated that regenerative mucosa was growing around the ulcer and that the ulcer was gradually diminishing in size (Figure $\mathbf{1} \mathbf{e}, \mathbf{f}$ ).

If the blood vessel had been a real varix, an embolism produced by the moving polymer of N-butyl-2-cyanoacrylate could have arisen after the sclerotherapy $[1,2]$, so we used the stock solution of $\mathrm{N}$ butyl-2-cyanoacrylate without diluting it in lipiodol. The injected N-butyl-2-cyanoacrylate did not appear to move from the surface of the exposed blood vessel. It is therefore sometimes useful to use the stock solution of N-butyl-2-cyanoacrylate for hemostasis of bleeding from a giant exposed blood vessel.

Endoscopy_UCTN_Code_TTT_1AO_2AD

\author{
K. Kurokohchii, ${ }^{1,2}$ T. Maeta ${ }^{2}$, T. Ohgi ${ }^{1,2}$, \\ M. Ono ${ }^{1,2}$, A. Yoshitake ${ }^{1,2}$, T. Yachida ${ }^{1,2}$, \\ M. Yoshida², Y. Mori² ${ }^{2}$ F. Kohi², \\ S. Kuriyama ${ }^{1}$ \\ ${ }^{1}$ Department of Gastroenterology and \\ Neurology, Kagawa University School of \\ Medicine, Kita-gun, Kagawa, Japan \\ 2 Department of Gastroenterolgy, KKR Ta- \\ kamatsu Hospital, Takamatsu, Kagawa, \\ Japan.
}

\section{References}

${ }^{1}$ Gallet B, Zemour G, Saudemont JP et al. Echocardiographic demonstration of intracardiac glue after endoscopic obturation of gastroesophageal varices. J Am Soc Echocardiogr 1995; 8: 759-761

2 Tan YM, Goh KL, Kamarulzaman A et al. Multiple systemic embolisms with septicemia after gastric variceal obliteration with cyanoacrylate. Gastrointest Endosc 2002; 55: $276-278$

\section{Corresponding author}

\section{S. Kuriyama, MD}

Department of Gastroenterology

and Neurology

Kagawa University School of Medicine 1750-1 Ikenobe

Miki-cho

Kita-gun

Kagawa 761-0793

Japan

Fax: +81-87-891-2158

Email: skuriyam@med.kagawa-u.ac.jp 